EXTENDED REPORT

\title{
Epidemiology of the optic nerve grey crescent in the Reykjavik Eye Study
}

\author{
O Jonsson, K F Damii, F Jonasson, A Arnarsson, T Eysteinsson, H Sasaki, K Sasaki
}

See end of article for authors' affiliations

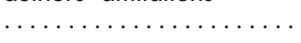

Correspondence to: Karim F Damii, University of Ottawa Eye Institute, 501 Smyth Road, Ottawa, Ontario, Canada K1H 8L6; kdamii@ohri.ca

Accepted for publication 10 May 2004
Br J Ophthalmol 2005;89:36-39. doi: 10.1136/bjo.2004.044305

Aim: To establish the epidemiology of the grey crescent in a white population within the age range most susceptible to glaucoma.

Methods: Bruce Shields was first to use this term to describe a localised, physiological pigmentation of the optic nerve neuroretinal rim tissue that is distinct from peripapillary pigmentation. An experienced glaucomatologist (KFD) evaluated stereofundus photographs of the participants of the Reykjavik Eye Study (RES) - a random sample from the national population census including people 50 years and older. 1012 right eyes could be evaluated for grey crescent.

Results: The prevalence of grey crescent in the right eyes was $22.0 \%(95 \% \mathrm{Cl} 10$ to 25$)$. It was more commonly found in women (27.0\%: 95\% Cl 23 to 30 ) than in men (17.0\%: 95\% Cl 14 to 21 ), and was most often located temporally $(36.9 \%), 360^{\circ}(15.9 \%)$, or nasally $(15.4 \%)$. The spherical equivalent was +1.30 dioptres (D) for those with and $+0.80 \mathrm{D}$ for those without grey crescent $(p=0.002)$, respectively. Vertical optic disc diameters were $0.203 \vee 0.195$ units $(p<0.001)$. There was no difference in the prevalence of grey crescent in glaucomatous or non-glaucomatous eyes (OR=1.05, 95\% $\mathrm{Cl} 0.49$ to 2.26). The prevalence of a grey crescent was inversely related to the prevalence of peripapillary atrophy $(\mathrm{p}=0.001)$.

Conclusions: The grey crescent needs to be recognised as a physiological variant in order to avoid falsely labelling eyes as having glaucomatous optic nerve damage.
I n 1980, Bruce Shields described a pigmented crescent of the optic disc which appeared to be located within the substance of the optic nerve head and termed this the grey crescent. ${ }^{1}$

The precise nature of this crescent is not known but it is thought to be a physiological variant and may represent an accumulation of melanocytes or melanin either on or within the neuroretinal rim tissue of the nerve, or possibly deeper within the lamina cribrosa (B Shields, personal communication). The importance of this crescent, unlike a peripapillary pigmented crescent, is that because of its location, it can obscure interpretation of the neuroretinal rim tissue when assessing the nerve for presence of glaucomatous damage. This may lead to an artefactually high assessment of the cup to disc ratio, or of localised rim tissue loss. Although Shields noted an association between presence of this crescent and myopia, diabetes, and black race in his clinic based study, the population epidemiology of this type of crescent has not been described to the best of our knowledge. In this paper we describe the prevalence of the grey crescent in white people within the age range most susceptible to glaucoma and its association with disc diameter, cup to disc ratio, peripapillary atrophy, intraocular pressure, visual acuity, and refraction.

\section{METHODS}

Permission for the study was granted by the university hospital ethics committee in Iceland and the Icelandic State Data Protection Commission. All subjects gave informed consent to their participation and all procedures adhered to the provisions of the Helsinki declaration. The Reykjavik Eye Study (RES) used a random sample from the national population census for Reykjavik. Included were people 50 years and older, an equal proportion for age and sex. A total of 1379 people qualified and of these, 1045 (461 males and 584 females) participated, a response rate of $75.8 \% .^{23}$ The participants filled in a questionnaire and had visual acuity measured on a Snellen chart, keratorefractometry (Nidek ARK 900), ${ }^{2}$ Scheimpflug photography of the anterior eye segment including the lens (Nidek EAS 1000), ${ }^{3}$ and stereo fundus photography (Nidek 3Dx/NM) ${ }^{4}$ with one photograph centred on the optic disc and the other on the macula. ${ }^{56}$ All participants were white. The photographs were evaluated in a masked fashion by an experienced glaucomatologist (KFD). Each stereo photograph was graded for quality ( $1-7$ with $1=$ poor and $7=$ excellent), horizontal and vertical disc diameters were measured, and nerve fibre layer, extension and location of peripapillary atrophy, horizontal and vertical cup to disc ratio, focal change of the optic nerve (notching and thinning of neuroretinal rim), disc haemorrhage, and presence and location of a grey crescent were noted. ${ }^{5}$ For measurement of optic disc diameters the white plastic diffuser from the stereo viewer was removed and picket circles 1203I were placed flush with the stereo viewer. The size of the disc was measured utilising a protocol that followed that of Klein et al. ${ }^{7}$ The disc measurements were recorded in arbitrary units and were corrected for refraction. No correction for camera or stereo viewer magnification was applied since these would be identical in all pictures. An anterior extension of sclera between the choroid, the so called scleral lip, appears commonly as a thin, even, white rim that marks the disc margin. ${ }^{8}$ A broader but incomplete and more irregular area of depigmentation is called the beta zone or chorioscleral crescent. ${ }^{9}$ It represents a retraction of retinal pigment epithelium from the disc margin, which is often associated with a thinning or absence of choroid next to the disc and exposure of the sclera. Zone alpha is a peripapillary crescent of increased pigmentation which may be peripheral to the beta zone or may be adjacent to the disc if the beta zone is absent. The presence of a grey crescent was defined by the occurrence of a pigmented crescent that appeared,

Abbreviations: PPA, peripapillary atrophy; RES, Reykjavik Eye Study 
utilising the stereo viewer, to be located on or within the neuroretinal rim tissue-that is, inside the scleral lip of the disc whereby the scleral lip had to be clearly visible peripheral to the crescent. If present, the location was determined depending on where the greatest extent was observed. Peripapillary atrophy (PPA) was noted when pigment and/ or atrophy could be seen outside the disc edge-that is, peripheral to the scleral lip. We did not use the alpha zone and beta zone grading of PPA because the alpha zone and/or beta zone can be present immediately outside the scleral lip in different locations for the same nerve, and because this grading system does not give a clear idea of radial or circumferential extent of PPA. We thus graded PPA according to radial extent and circumferential extent. The radial extent of PPA (that is, the width of PPA adjacent to the nerve) was graded as 0 for no atrophy, 1 for minimal atrophy, 2 for moderate atrophy, 3 for marked atrophy, and 4 for extensive atrophy. Circumferential extent (location) was recorded as clockwise extent of contiguous areas according to a clock face superimposed on the nerve. The total number of clock hours of PPA was also recorded. The definition of glaucoma followed the criteria outlined by Foster et $a l^{10}$ and is also summarised in detail in a review of glaucoma prevalence in the RES. ${ }^{5}$ The intraocular pressure was measured using the Nidek NT 2000 non-contact air puff tonometer. ${ }^{11}$ In this paper we describe the results for the right eyes. The results for the left eyes were similar.

Statistical calculation, including descriptive statistics, $t$ tests, phi coefficient, Fisher's exact test, and logistic regression, were done using SPSS 11.0 (SPSS Inc, Chicago, IL, USA).

\section{RESULTS}

\section{Quality of photographs}

Quality grade 1 and 2 were disregarded for this analysis. Of the 1045 people examined fundus photographs of 1012 right eyes were available and of sufficient quality for analysis

\section{Grey crescent}

The prevalence of grey crescent by age and right eyes can be seen in table 1. A grey crescent was found in 226 right eyes, giving an overall prevalence in this study population of $22.0 \%$ (95\% CI 10 to 25 ) for right eyes. Of those with grey crescent in the right eye, $64.2 \%$ (95\% CI 58 to 70 ) were women and $35.8 \%$ (95\% CI 30 to 42 ) men. In both calculations, females turned out to be significantly more likely to have grey crescent than men $(p=0.001)$. Looking at either eye, the
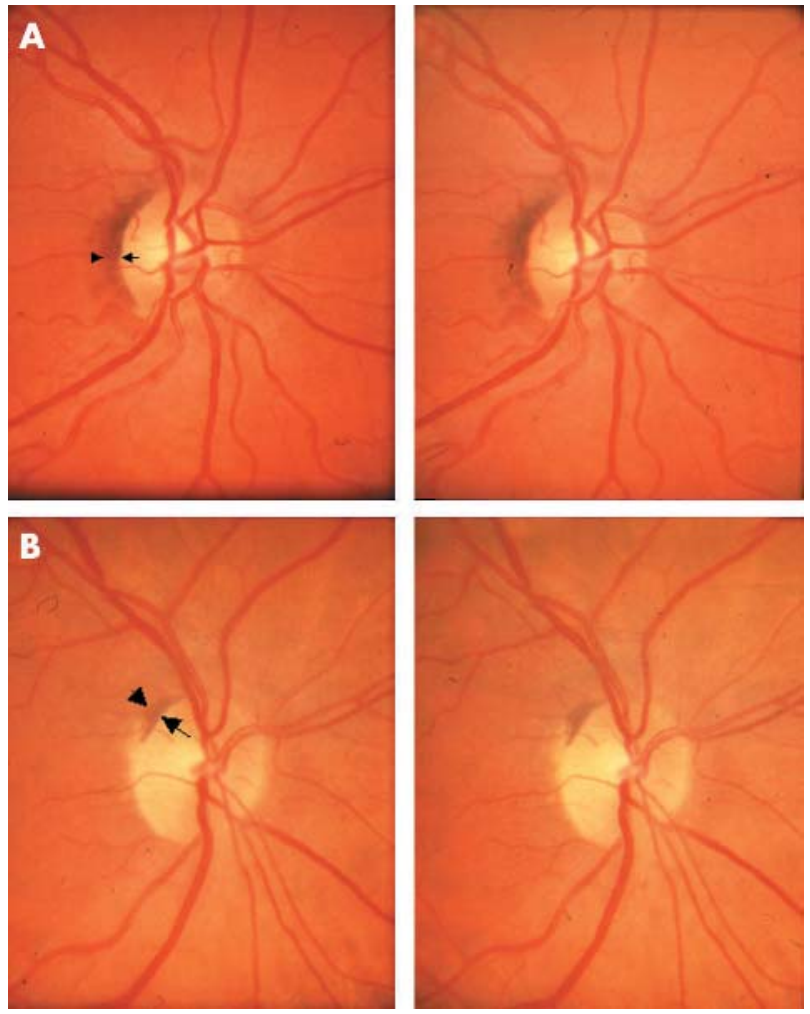

Figure 1 Examples of grey crescents located temporally (A) and superotemporally (B). Arrows point to inner edge of grey crescent and arrowheads point to outer edge of disc (that is, the scleral lip).

prevalence of grey crescent among men was $17.0 \%$ (95\% CI 14 to 21 ) compared to $27.0 \%$ (95\% CI 23 to 30 ) among women. Of those who had grey crescent in their right eye, $57.9 \%$ were bilaterally affected. Age did not affect the probability of presence of a grey crescent.

The grey crescent was most often located temporally (table 2). The grey crescent was located only temporally in $36.9 \%$ of instances (fig lA) but occurred there as well as in other quadrants combined in $39.7 \%$ of those affected (fig 1B illustrates a superotemporal grey crescent). In $15.4 \%$ a grey crescent was found only nasally and in $15.9 \%$ it was found $360^{\circ}$. A grey crescent was rarely found in the inferior $(1.4 \%)$ or superior ( $1.4 \%)$ quadrants only.

Table 1 Prevalence of grey crescent by age, sex, for right eyes

\begin{tabular}{lccl}
\hline Age/years & Number at risk & Prevalence $(\mathbf{n})$ & $\%$ Prevalence $(95 \% \mathrm{Cl})$ \\
\hline Males & 164 & & $15 \%(9$ to 20$)$ \\
$50-59$ & 142 & 24 & $17 \%(11$ to 23$)$ \\
$60-69$ & 130 & 24 & $19 \%(12$ to 26$)$ \\
$70-79$ & 31 & 8 & $26 \%(9$ to 42$)$ \\
$80+$ & 467 & 81 & $17 \%(14$ to 21$)$ \\
Total & 187 & 45 & $24 \%(18$ to 30$)$ \\
Females & 205 & 62 & $30 \%(24$ to 37$)$ \\
$50-59$ & 113 & 22 & $19 \%(12$ to 27$)$ \\
$60-69$ & 40 & 16 & $40 \%(24$ to 56$)$ \\
$70-79$ & 545 & 145 & $27 \%(23$ to 30$)$ \\
$80+$ & & & \\
Total & 351 & 69 & $20 \%(15$ to 24$)$ \\
Sexes combined & 347 & 86 & $25 \%(20$ to 29$)$ \\
$50-59$ & 243 & 47 & $19 \%(14$ to 24$)$ \\
$60-69$ & 71 & 24 & $22 \%(23$ to 45$)$ \\
$70-79$ & 1012 & 226 & \\
$80+$ & & & \\
Total & & & \\
\hline & &
\end{tabular}


Table 2 Locations of grey crescent (GC) and peripapillary atrophy (PPA), right eyes

\begin{tabular}{lll}
\hline Location & GC $(\%)$ & PPA $(\%)$ \\
\hline Temporal only & 36.9 & 41.9 \\
Nasal only & 15.4 & 0.4 \\
Superior only & 1.4 & 0.3 \\
Inferior only & 1.4 & 0.7 \\
$360^{\circ}$ & 15.9 & 38.1 \\
Superior-nasal & 2.3 & 1.1 \\
Superior-temporal & 10.7 & 4.4 \\
Inferior-nasal & 1.9 & 0.9 \\
Inferior-temporal & 8.9 & 4.4 \\
Inferior 180 degrees & 2.3 & 2.0 \\
Superior 180 degrees & 1.9 & 0.3 \\
Nasal 180 degrees & 0.0 & 1.8 \\
Temporal 180 degrees & 0.0 & 3.5 \\
Intermittent & 0.9 & 0.2 \\
Total & 100 & 100 \\
\hline
\end{tabular}

\section{Optic nerve characteristics}

When comparing optic disc diameters in those with and without a grey crescent, there was a significant difference in both vertical and horizontal disc diameters. Vertical disc diameter in grey crescent was 0.203 (SD 0.023) units $v 0.195$ (SD 0.021) units in eyes without grey crescent $(\mathrm{p}<0.001)$. Horizontal disc diameters in grey crescent were 0.194 (SD 0.021 ) units $v 0.186$ (SD 0.021) units in eyes without grey crescent $(\mathrm{p}<0.001)$. There was also a slight but statistically significant difference in C/D ratio between those with and without grey crescent. The mean vertical C/D ratio for those with grey crescent was 0.5 (SD 0.2) and for those without grey crescent it was 0.45 (SD 0.22 ), which is a statistically significant difference $(\mathrm{p}=0.002)$. The mean horizontal C/D ratio for those with grey crescent was 0.49 (SD 0.19) and for those without grey crescent it was 0.44 (SD 0.21 ), which also reaches statistical significance $(p=0.002)$. Definite glaucomatous optic nerve changes were noted in $5.0 \%$ of those with grey crescent and in $4.7 \%$ of those without grey crescent. This difference is not statistically significant, OR $=1.05$ (95\% CI 0.49 to 2.26 ). There was no relation between the appearance of grey crescent and focal notching of the optic nerves.

\section{Peripapillary atrophy}

PPA was evident in 653 eyes, which is $65 \%$. Peripapillary atrophy was found to be common in all age groups, the prevalence increasing with age $(\mathrm{OR}=1.04$ /year, 95\% CI 1.02 to $1.05, \mathrm{p}<0.001$ ) (table 3 ). The radial extent of PPA was usually minimal (grade 1), sometimes moderate (grade 2) and rarely marked or extensive (grades 3 and 4, respectively). Of those without PPA 30\% had grey crescent. Of those with PPA present in one quadrant $19 \%$ had grey crescent, with PPA in two quadrants $15 \%$ had grey crescent, with PPA in three quadrants $10 \%$ had grey crescent, and none of those with PPA in four quadrants had grey crescent. Calculation of the phi coefficient revealed a statistically significant inverse relation between circumferential extent of PPA and prevalence of grey crescent $(\mathrm{phi}=0.166, \mathrm{p}=0.001)$.

\section{Intraocular pressure, visual acuity, and refraction}

The mean IOP was the same for those with and without grey crescent, (15.5 (SD 3.4) $\mathrm{mm} \mathrm{Hg}$ and 15.5 (SD 3.2) $\mathrm{mm} \mathrm{Hg,}$ respectively). There was no difference in mean visual acuity as measured by Snellen chart for those with and without grey crescent $(p=0.7)$. The mean spherical equivalent of those with grey crescent was $+1.3 \mathrm{D}$ (SD 2.07) and +0.8 D (SD 2.25) for those without grey crescent. This difference was statistically significant $(\mathrm{p}=0.002)$.

\section{DISCUSSION}

The grey crescent is a localised physiological pigmentation of the optic nerve (probably on or within the neuroretinal rim tissue) that is distinct from peripapillary pigmentation. It needs to be recognised as being part of the disc tissue in order to avoid the false perception of localised neuroretinal rim thinning and hence glaucomatous disc damage. This study describes for the first time to our knowledge, the epidemiology of grey crescent in a white population. We are unaware of any previous studies that report prevalence of the grey crescent. Our study demonstrates that the grey crescent is a common variant in white people 50 years and older. The grey crescent is more common in women than in men and is associated with hyperopic eyes, larger optic discs as well as cups, and its prevalence is inversely related to the presence of peripapillary atrophy. The larger cup size may be associated with larger discs in those with grey crescent. ${ }^{12}$ In his clinic based study in North Carolina, Shields ${ }^{1}$ found an association between grey crescent and myopia and diabetes. This was not confirmed in our population based study. We found grey crescent associated with hyperopia and no association to diabetes or other systemic diseases. Shields also pointed out that the grey crescent was related to black race; however, our study shows that the phenomenon is also prevalent among white people. Grey crescent is easily identified in most instances, though at times it may be more difficult and may make estimates of the neuroretinal rim difficult.

The aetiology of the grey crescent is unclear. It may be pigment within cells-for example, melanocytes, retinal pigment epithelial cells, or other cells within the nerve substance, or it may represent free pigment granules within the neuroretinal rim tissue or perhaps deeper within the lamina cribrosa. There are variations in the peripapillary pigmentation that can sometimes interfere with the interpretation of the optic nerve head. Among them are the alpha zone and zone beta, with the latter possibly representing a malposition of the embryonic fold, either a double layer or irregularity of retinal pigment epithelium. ${ }^{13}$ We did not grade PPA according to the alpha zone or beta zone but instead chose to characterise radial and circumferential extent of PPA. Unlike the peripapillary areas of pigment-for example, zone beta, the grey crescent appears to be within the substance of the optic nerve head and inside the scleral lip (disc edge). The fluorescein angiographic

Table 3 Peripapillary atrophy by age and right eye (PPA by grades, right eyes)

\begin{tabular}{|c|c|c|c|c|c|c|c|}
\hline Age (years) & $\begin{array}{l}\text { Number at } \\
\text { risk }\end{array}$ & Prevalence (n) & \% Prevalence $(95 \% \mathrm{Cl})$ & Grade 1 & Grade 2 & Grade 3 & Grade 4 \\
\hline \multicolumn{8}{|c|}{ Sexes combined } \\
\hline $50-59$ & 351 & 202 & $58 \%$ (52 to 63 ) & $42 \%$ & $14 \%$ & $2 \%$ & $1 \%$ \\
\hline 60-69 & 347 & 217 & $63 \%$ (57 to 68 ) & $43 \%$ & $13 \%$ & $6 \%$ & $1 \%$ \\
\hline 70-79 & 242 & 174 & $72 \%$ (66 to 78 ) & $45 \%$ & $19 \%$ & $6 \%$ & $1 \%$ \\
\hline $80+$ & 71 & 60 & $85 \%$ (76 to 93 ) & $39 \%$ & $24 \%$ & $17 \%$ & $4 \%$ \\
\hline Total & 1011 & 653 & $65 \%$ (63 to 69$)$ & & & & \\
\hline
\end{tabular}


findings that superficial vessels of the optic nerve head extend over the grey crescent, while the deep fluorescein of the nerve head is obscured, to a variable degrees, by the crescent ${ }^{1}$ supports this evidence. Unlike peripapillary atrophy, which increases in prevalence with age and is associated with glaucoma, ${ }^{14}$ the grey crescent does not appear to be associated with either age or glaucoma in the RES. ${ }^{5}$

The importance of the grey crescent is that because of its location within the disc inside the scleral lip, the grey crescent can in some instances make the neural rim look thinner than it actually is giving the false impression of a focal thinning or atrophy of the optic nerve. This can lead to a false diagnosis of glaucomatous damage. Our finding that grey crescent is common in white individuals from the RES aged over 50 years, makes it important to keep in mind when evaluating optic nerves for glaucomatous damage in a white population.

\section{ACKNOWLEDGEMENTS}

This work was supported by the Icelandic Research Council and the St Joseph's Hospital Research Fund. Drs L Wang and R Munger provided assistance in calculation of corrected disc diameters.

\section{Authors' affiliations}

O Jonsson, Department of Ophthalmology, Sahlgrenska University Hospital, Gothenburg, Sweden

K F Damii, University of Ottawa Eye Institute and Ottawa Health Research Institute, Ottawa, Canada

F Jonasson, A Arnarsson, Department of Ophthalmology, University of Iceland, Reykjavik, Iceland

T Eysteinsson, Department of Physiology, University of Iceland, Reykjavik, Iceland

H Sasaki, K Sasaki, Kanazawa Medical University, Uchinada, Japan

\section{REFERENCES}

1 Shields MB. Grey crescent in the optic nerve head. Am J Ophthalmol 1980;89:238-44

2 Gudmundsdottir $\mathbf{E}$, Jonasson $F$, Jonsson $V$, et al. "With the rule" astigmatism is not the rule in the elderly. Reykjavik Eye Study: a population based study of refraction and visual acuity in citizens of Reykjavik 50 years and older. Acta Ophthalmol Scand 2000;78:642-6.

3 Katoh N, Jonasson F, Sasaki H, et al. Cortical lens opacification in Iceland. Risk factors analysis. Reykjavik Eye Study. Acta Ophthalmol Scand 2001;79:154-9.

4 Greenfield DS, Zacharia P, Schuman JS. Comparison of Nidek 3Dx and Donaldson Simultaneus Stereoscopic Disc Photography. Am J Ophthalmol 1993; 116:741-7.

5 Jonasson F, Damii KF, Arnarsson A, et al. Prevalence of open angle glaucoma in Iceland: Reykjavik Eye Study. Eye 2003;17:747-53.

6 Jonasson F, Arnarsson A, Sasaki H, et al. The prevalence of age-related maculopathy in Iceland. Reykjavik Eye Study. Arch Ophthalmol 2003; 121:379-85.

7 Klein BEK, Moss SE, Magli YL, et al. Optic disc cupping as clinically estimated from photographs. Ophthalmology 1987;94:1481-3.

8 Rockwood EJ, Anderson DR. Acquired peripapillary changes and progression in glaucoma. Graefes Arch Klin Exp Ophthalmol 1988;226:510-15.

9 Tuulonen A, Jonas JB, Välimäki S, et al. Interobserver variation in the measurements of peripapillary atrophy in glaucoma. Ophthalmology 1996; 103:535-41.

10 Foster PJ, Buhrmann R, Quigley HA, et al. The definition and classification of glaucoma in prevalence surveys. $\mathrm{Br} J$ Ophthalmol 2002;86:238-42.

11 Eysteinsson T, Jonasson F, Sasaki H, et al. Central corneal thickness, radius of the corneal curvature and intra ocular pressure in normal subjects using noncontact techniques: Reykjavik Eye Study. Acta Ophthalmol Scand 2002;80:11-15.

12 Wang L, Damji KF, Munger R, et al. Increased disk size in glaucomatous eyes versus normal eyes in the Reykjavik Eye Study. Am J Ophthalmol 2003; 135:226-8.

13 Shields MB. Textbook of glaucoma, 4th ed. Philadelphia: Lippincott Williams and Wilkins, 1997.

14 Jonas JB, Budde WM, Panda-Jonas S. Ophthalmoscopic evaluation of the optic nerve head. Surv Ophthalmol 1999;43:293-320. 